\title{
Role of positron-emission tomography/ computed tomography (PET/CT) in breast cancer
}

\author{
Mohamed Shawky ${ }^{1}$, Zeinab Abd Elaziz Ali ${ }^{1}$, Dina Hamdy Hashem ${ }^{2 *}$ and Mohamed Houseni ${ }^{2}$
}

\begin{abstract}
Background: Breast cancer is the most common cancer type in women and the most common cause of deaths in women worldwide.

FDG-PET/CT was used in the early stage, estimation of the therapeutic response, revelation of recurrent disease, and distal metastasis. However, with the increasing use of FDG PET/CT, sites of accelerated activity have been occasionally found out in unexpected locations which may not correlate with the patient's clinical history or the expected propagation of the primary malignancy.

The aim of this study is to detect the diagnostic value of PET/CT in breast cancer patients; comparing PET/CT performance with that of contrast-enhanced CT in diagnosis of breast cancer and distant metastasis.

Results: The study included 30 female patients with breast cancer, mean age 53.56 years \pm 10.64 (SD), age range 33-73 years. PET/CT detect contralateral breast affection in 2 patients (6.7\%), and distant metastasis was seen on PET/CT. Sites of distant metastasis included the bone $(n=12)$, axillary lymph nodes $(n=11)$, cervical lymph nodes $(n=6)$, mediastinal lymph nodes $(n=12)$, abdominal lymph nodes $(n=8)$, liver $(n=5)$, lung $(n=11)$, and other visceral sites metastasis $(n=9$ ). PET/CT detected breast lesions with a sensitivity of $100 \%$ and specificity of $95.4 \%$. In contrast, the sensitivity and specificity of CT alone were $81.2 \%$ and $90.4 \%$, respectively.

Conclusion: PET/CT has superiority over CT alone in revelation of breast lesions and distant metastases.

Keywords: Breast cancer, CT, Metastasis, PET/CT
\end{abstract}

\section{Background}

Breast cancer is the most common cancer type and the most common cause of deaths in women worldwide [1].

Many risk factors are well-known; however, the exact causes of breast cancer have not been identified. Family history of breast cancer, for example, is a well-known factor that increases risk by a factor of two or three. Also, mutations such as BRCA (1 and 2) and p53 are considered to convey a very strong risk of developing the disease [2].

\footnotetext{
* Correspondence: Dr.dina_hh20@yahoo.com

${ }^{2}$ Department of Radiodiagnosis, National Liver Institute, Menoufia University, shibin el kom, menoufia goverment 32511, Egypt

Full list of author information is available at the end of the article
}

FDG-PET/CT is used in the early stage, estimation of the therapeutic response, revelation of recurrent disease, and distal metastasis. However, with the increasing use of FDG PET/CT, sites of accelerated activity have been occasionally found out in unexpected locations which may not correlate with the patient's clinical history or the expected propagation of the primary malignancy [3].

Positron emission tomography (PET) with or without integrated computed tomography (CT) using 18F-fluorodeoxyglucose (FDG) is based on the principle of increased glucose metabolism in malignant tumors and has been investigated frequently in breast cancer [4].

\section{Springer Open}

(c) The Author(s). 2020 Open Access This article is licensed under a Creative Commons Attribution 4.0 International License, which permits use, sharing, adaptation, distribution and reproduction in any medium or format, as long as you give appropriate credit to the original author(s) and the source, provide a link to the Creative Commons licence, and indicate if changes were made. The images or other third party material in this article are included in the article's Creative Commons licence, unless indicated otherwise in a credit line to the material. If material is not included in the article's Creative Commons licence and your intended use is not permitted by statutory regulation or exceeds the permitted use, you will need to obtain permission directly from the copyright holder. To view a copy of this licence, visit http://creativecommons.org/licenses/by/4.0/. 
The increased metabolic activity in oncological cells is the key point when thinking about the importance of FDG-PET imaging for detection of malignant tumors [5].

Many researches have demonstrated the advantages of PET/CT over conventional imaging modalities in detecting lymph nodal metastasis, including infraclavicular, supraclavicular, and internal mammary lymph nodes. Detection of such disease involvement may upstage patients to stage III impact prognosis and may change the patient's initial treatment strategy to include for example, resection of infraclavicular nodes or radiotherapy to extraaxillary nodes [6].

Estimating the sensitivity and specificity of contrastenhanced CT (CECT) and PET/CT for re-staging of patients with dubious recurrence of breast cancer and complemented that FDG-PET/CT can ameliorate staging and therefore impact clinical management in patients with suspected cancer breast recurrence and distant metastatic disease [7].

The aim of this study was to detect the diagnostic power of PET/CT in breast cancer patients. We compared the performance of PET/CT with that of CECT in detected breast cancer and distant metastasis.

\section{Methods}

This is a prospective study carried out at the authors' institution from January 2016 to December 2017. It included 30 female patients of ages ranged from 33-73 years with mean age of 53.5 years. All patients had pathologically proven breast cancer.

\section{Ethical consideration}

Consent was taken from patients or their relatives before doing PET/CT, and they had the right to refuse at any time. The study was approved by the Research Ethics Committee of our Institute and the Research Ethics Committee of the Faculty of Medicine.

In this prospective study, 30 female patients who were diagnosed as breast cancer and started treatment then came to do whole body PET-CT and contrast-enhanced $\mathrm{CT}$ to assess the breast cancer and search for metastasis and recurrence. All patients were followed up for period range between 6 and 12 months.

All patients were subjected to the following: full history taking and laboratory investigation as blood glucose and serum creatinine.

All exams were performed using a Siemens Biograph dual source mCT20 Excel PET/CT, equipped with 128 slices CT.

The patients were submitted for the study and reviewed against inclusion and exclusion criteria as follows.
Inclusion criteria were all cases of histologically proven breast cancer. Some cases were positive operative history (modified radical mastectomy, simple mastectomy, and lumpectomy). The study included patients who received chemotherapy with last cycle for more than 3 weeks and radiotherapy with last session for more than 2 months.

Exclusion criteria were early post-operative cases, uncontrolled diabetic patients, and patients with impaired renal function.

All patients underwent 18F-FDG PET/CT examinations using a dedicated PET/CT scanner equipped with a 128-slice CT system (Siemens, Biograph mCT 128; Siemens Medical Solutions, Knoxville, USA). Patients fasted for about $6 \mathrm{~h}$ before the injection time. Serum glucose levels were measured before injection to confirm euglycemia (blood glucose level $<160 \mathrm{mg} /$ $\mathrm{dL}$ ). Then, $370-550 \mathrm{MBq}$ of $18 \mathrm{~F}-\mathrm{FDG}$ was injected with a saline infusion in the antecubital vein depending on the weight. Patients were asked to drink 750 $\mathrm{mL}$ of $5 \%$ mannitol as a negative oral contrast during the uptake phase. Approximately after $60 \mathrm{~min}$ from injection, PET/CT scans were performed. Scanning was performed in a supine position with patient's arms rested above the head.

A low-dose CT scan, for attenuation correction and anatomical mapping. The acquisition was performed in helical mode from the skull base to midthigh, using $150 \mathrm{kV}, 50 \mathrm{mAs}$, and a $512 \times 512$ matrix size, acquiring a field of view (FOV) of 780 $\mathrm{mm}$ in about $20 \mathrm{~s}$.

The emission PET scan was acquired, after the low dose CT, in a three-dimensional mode (3D) from the skull base to mid-thigh. Imaging was performed, using sequential overlapping bed positions with the acquisition time of $3 \mathrm{~min}$ per bed position. The number of bed positions depends on the patient's height with one-slice overlap at the borders of the FOV. Deadtime correction and online delayed coincidence subtraction were used to correct for random coincidences. The low-dose CT images were rescaled to produce attenuation correction values for the PET scan. Image reconstruction was based on point-spread function (PSF) together with time of flight (TOF) (Siemens "ultra-HD.PET"; iterations, 2; subsets, 21). The projection data were reconstructed by $3-\mathrm{mm}$ slice thickness. Gaussian filter (FWHM, $2 \mathrm{~mm}$ ) was used for the reconstructed data. Finally, the low-dose CT was reconstructed using a convolution kernel B20f body filter.

Following PET imaging, volumetric contrast-enhanced CT scanning was performed from the skull base to midthigh on the same PET/CT machine using the helical 120-slice multi-detector CT system of the PET/CT 
scanner. Intra-venous contrast was given at a rate of $1.5 \mathrm{ml} / \mathrm{kg}$ with overall dose ranging from 80 to 100 $\mathrm{ml}$. The CT acquisitions were performed using a 1.2pitch and $20 \times 0.6 \mathrm{~mm}$ collimation for detector configuration. The gantry rotation time was $0.5 \mathrm{~s}$, field of view of $78 \mathrm{~cm}$, and reconstruction matrix of $512 \times$ 512. Automated exposure control was applied for all patients (CARE Right, Siemens Medical Solutions, Forchheim, Germany) with $120 \mathrm{kV}$ tube potential and the average $\mathrm{mAs}$ of 150 . Trans-axial images were reconstructed at $1.5-\mathrm{mm}$ slice thickness in $1-\mathrm{mm}$ increment using a convolution kernel B20f body filter. For multi-planar evaluation, coronal and sagittal images with a $1.5-\mathrm{mm}$ slice thickness in 1-mm increment were reconstructed.

Images were reconstructed and viewed on workstation which provided multi-planar reformatted PET, CT, and fused PET/CT images as well as MIP PET images in video mode.

\section{Interpretation of images}

Images were assessed by two radiologist, one of them with 10 years' experience in PET/CT analysis, the other with 3 years of experience in PET/CT. Conjoined, both were aware of clinical data. Both radiologists revised the images together till full agreement.

Different methods were used for assessment of radiotracer uptake by normal and pathologic tissues; analysis of PET images was via visual and semi-quantitative assessment (SUV max measurement). Active lesions were recorded at areas of high FDG uptake. SUV max was measured at each lesion and compared to background activity. The standard background activity was measured at the liver (right lobe). In patients having diseased liver, the background activity was measured at the mediastinal blood pool.

\section{Reference standard}

The diagnosis of the breast cancer, recurrence, and distant metastasis was made after histo-pathological analysis and clinical and imaging follow-up.

\section{Statistical analysis of the data}

Data were fed to the computer and analyzed using the IBM SPSS software package version 20.0. (Armonk, NY: IBM Corp). Qualitative data were described using number and percent. The Kolmogorov-Smirnov test was used to verify the normality of distribution; quantitative data were described using range (minimum and maximum), mean, standard deviation, and median. Significance of the obtained results was judged at the $5 \%$ level.

\section{Results}

The study included 30 female patients with breast cancer, the mean age \pm standard deviation (SD) $53.56 \pm$ 10.64 years, age range $33-73$ years.

\section{Value of PET/CT scan}

Detection of the contralateral breast affection in 2 patients $(6.7 \%)$ and distant metastasis were visualized on PET/CT (Fig. 1). Sites of distant metastasis included the bone $(n=12)$ (Fig. 1b2), axillary lymph nodes $(n=11)$ (Fig. 1a2), cervical lymph nodes $(n=6)$, mediastinal lymph nodes $(n=12)$, abdominal lymph nodes $(n=8)$, liver $(n=5)$, lung $(n=11)$, and other visceral sites metastasis $(n=9)$ (Table 1$)$.

\section{Comparison between PET/CT and contrast-enhanced CT to detect breast lesion of both breast in $\mathbf{3 0}$ patients with breast cancer}

We compare the performance of both PET/CT and contrast-enhanced CT techniques to detect breast lesion of both breast in 30 patients with breast cancer. The true total positive cases by PET/CT were 16 patients, 2 false positive patients, 42 true negative patients, and no false negative case (Table 2).

The sensitivity, specificity, accuracy, and positive and negative predictive value of FDG-PET/CT and contrast-enhanced $\mathrm{CT}$ in all breast lesion cases were as follows: the sensitivity of PET/CT examinations was $100 \%$, specificity was $95.4 \%$, positive and negative predictive values of $\mathrm{PET} / \mathrm{CT}$ were $88.9 \%$ and $100 \%$ respectively, while the sensitivity of CT alone was $81.2 \%$, specificity was $90 \%$, positive and negative predictive values were $76.4 \%$ and $93 \%$ respectively (Fig. 2a2) (Table 3).

\section{Comparison between PET/CT and contrast-enhanced CT to detect distant metastasis in $\mathbf{3 0}$ patients with breast cancer}

Currently, the performance of both PET/CT and contrast enhanced CT techniques were compared to detect distant metastasis in 30 patients with breast cancer. The true total positive cases by PET/CT were 20 lymph nodes, 11 bone lesions, 11 pulmonary nodules, 4 hepatic lesions, and 8 other visceral metastases. The false negative cases by PET/CT were 1 bone lesion, 1 hepatic lesion, and 1 other visceral metastases (Fig. 2b2). The false positive cases by PET/CT were 2 lymph nodes and 2 other visceral metastases. The true negative cases by PET/CT were 8 lymph nodes, 18 bone lesions, 19 pulmonary nodules, 25 hepatic lesions (Fig. 2c2), and 19 other visceral metastases (Table 4).

The sensitivity, specificity, accuracy, and positive and negative predictive value of FDG-PET/CT and contrast- 

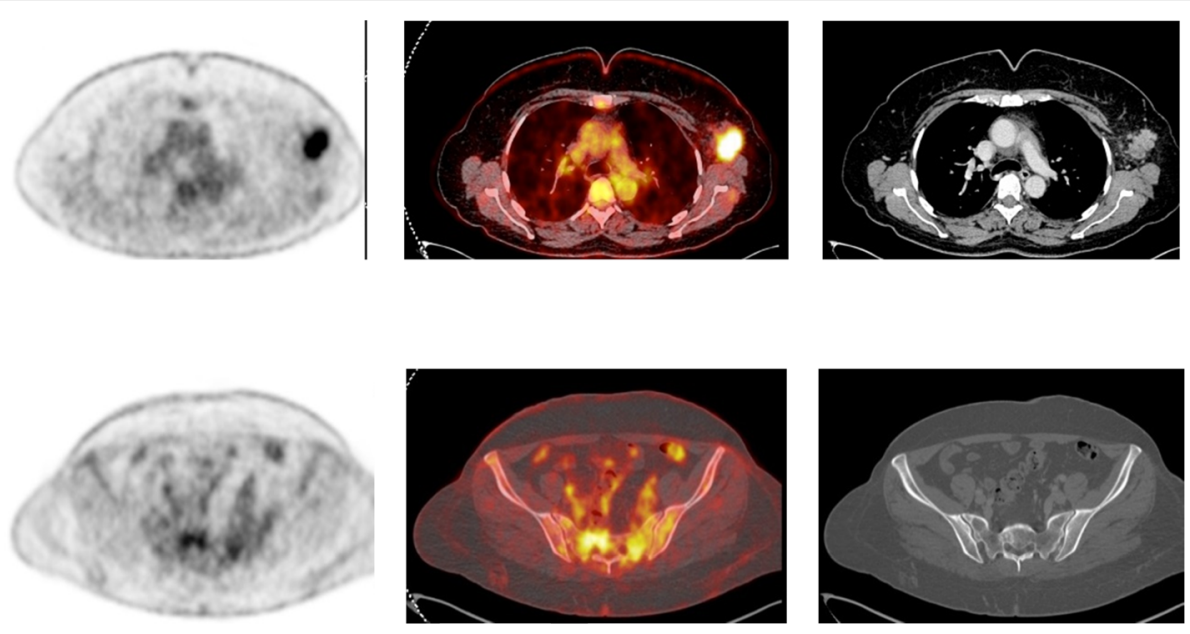

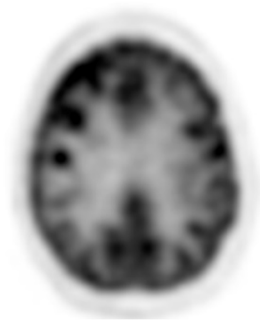

(C1) PET

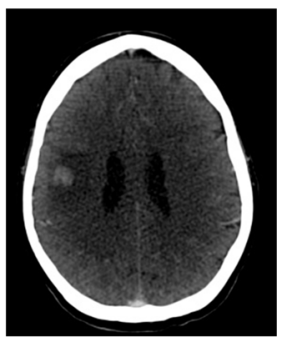

(C2) CECT

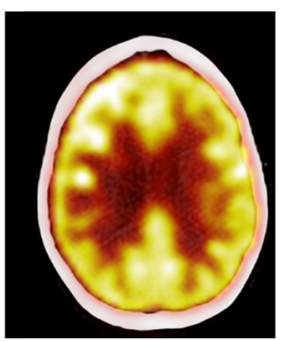

(C3) Fused PET/CT

Fig. 1 a $(\mathbf{1}, \mathbf{2}, \mathbf{3})$ PET, fused PET $C T$, and CECT axial images show FDG-avid heterogeneously enhancing left axillary tail breast mass measuring $4 \times$ $2.5 \mathrm{~cm}$ with SUV max 9. No evidence of chest wall invasion or overlying skin thickness. $\mathbf{b}(\mathbf{1}, \mathbf{2}, \mathbf{3})$ PET, fused PET CT, and CECT axial images show increased FDG uptake corresponding to right sacral sclerotic bone lesion with SUVmax 7.6. c $(\mathbf{1}, \mathbf{2}, \mathbf{3})$ PET, CECT, and fused PET CT axial images show increased FDG uptake corresponding to right fronto-parietal subcortical-enhancing lesion measuring about $1 \mathrm{~cm}$ with SUVmax20. Impression: Metabolically active left axillary tail breast neoplasm with metastatic bone marrow and brain deposits

enhanced CT in lymph nodes, bone, lung, liver, and other visceral metastasis (Table 5)

\section{Discussion}

Breast cancer is the most frequently diagnosed cancer in women. It affects more than 1 million women worldwide [8].

This study is prospective evaluation of patients suffering from breast cancers.

It included 30 female patients with age range 33-73. All patients were known to have breast cancers and were referred for whole body PET-CT scans.

In this study, PET/CT has detected lesions in the contralateral breast in $2 / 30$ patients $(6.7 \%)$ that was not detected by CT study providing advantage of fused PET/CT study over CT study alone; this comes in agreement with Ingrid et al. [9] who stated that PET/CT depicted additional lesions not visible at CT study alone and with Lim et al. [10] who stated that the advantage of PET/CT in front of other imaging modalities is the capability to discovered unsuspected distant metastases during a single whole-body examination.

In this study, 12/30 cases (40\%) were diagnosed with bone metastases. Osteoblastic, osteolytic, and mixed bone metastases were detected by CT and PET/CT which comes in an agreement with Piva et al. [8] who stated that the skeleton is the most common site of distant metastases in breast cancer.

In current study, we detected metabolically active FDG avid bony metastases in $40 \%$ of cases with 7 cases mixed lytic/sclerotic lesions, 3 with marrowbased lesion, and 2 with lytic lesions. Also, we found patients (3/30) who present a bony lesion with only marrow activity and no corresponding CT changes (marrow-based early metastatic deposit). This goes in concordance with the results of previous study done by Wafaie et al. [11] who stated that PET can 
Table 1 Contralateral breast affectation, lymph nodes, and other metastasis among breast cancer patient by PET/CT

\begin{tabular}{lll}
\hline & No. & $\%$ \\
\hline Contralateral breast affectation & 2 & 6.7 \\
LN affected & 11 & 36.7 \\
Axillary & 6 & 20.0 \\
Cervical & 12 & 40.0 \\
Mediastinal & 8 & 26.7 \\
Abdominal & 12 & 40.0 \\
Bone metastasis & 5 & 16.7 \\
Hepatic metastasis & 11 & 36.7 \\
Pulmonary metastasis & 9 & 30.0 \\
Other sites metastasis & 1 & 3.3 \\
Adrenal gland & 1 & 3.3 \\
Ovary & 2 & 6.7 \\
Brain & 1 & 3.3 \\
Peritoneal nodules & 2 & 6.7 \\
Subcutaneous nodules & 2 & 6.7 \\
Pleura & &
\end{tabular}

recognize bone marrow-based metastases early and in the absence of morphologic changes on CT images; this is improving CT sensitivity.

Current results came in an agreement with the findings of Wafaie et al. [11] who stated that fused PET/CT was highly efficient in the evaluation of skeletal metastases with superior performance in detection of early bone marrow infiltration not apparent on $\mathrm{CT}$ and resolution of metabolic activity before definite signs of complete healing on $\mathrm{CT}$.

The liver is the main visceral organ where metastases from breast cancer occur. The locoregional recurrence occurs early than liver metastases, and this is associated with a much worse prognosis [8].

This study depicted metabolically active hepatic metastatic deposits in (5/30) 16.7\%. In current study, patients with distant hepatic lesions (lesions were $>3$ $\mathrm{cm}$ in diameter) show positive results on PET-CT study; these results comes in agreement with Eubank et al. [12] who stated that the sensitivity of FDG PET

Table 2 Diagnostic performance of PET/CT and contrastenhanced CT to detect breast lesion in both breast of 30 cases with breast cancer

\begin{tabular}{|c|c|c|c|c|}
\hline \multicolumn{5}{|c|}{ Both breast lesion No (60) } \\
\hline & \multicolumn{2}{|c|}{ Cancer case $(n=16)$} & \multicolumn{2}{|c|}{ Non cancer case $(n=44)$} \\
\hline & True positive & $\overline{\text { False negative }}$ & False positive & True negative \\
\hline $\mathrm{PET} / \mathrm{CT}$ & 16 & 0 & 2 & 42 \\
\hline CT & 13 & 3 & 4 & 40 \\
\hline
\end{tabular}

is limited (25-43\%) for detection of metastatic lesions less than $1 \mathrm{~cm}$ in diameter.

This prospective study which includes 30 patients with breast cancer revealed the diagnostic power of PET/CT in breast cancer patients and compared the performance of PET/CT with that of enhanced CT alone in revelation of breast lesions, recurrence, and distant metastasis. The PET/CT interpretations were correct in 28/30 patients. Incorrect interpretations of PET/CT images occurred in 2 patients (two false positive cases). According to current results, the sensitivity of PET/ CT examinations was $100 \%$, specificity was $95.4 \%$, positive and negative predictive values of $\mathrm{PET} / \mathrm{CT}$ were $88.9 \%$ and $100 \%$, respectively, while the sensitivity of CT alone was $81.2 \%$, specificity was $90 \%$, positive and negative predictive values were $76.4 \%$ and $93 \%$ respectively. Reason for false positive results was increase FDG uptake corresponding to soft tissue thickening that presented total resolution in a progressed case on a follow-up study denoting its benign/inflammatory nature.

In this study, PET/CT has higher sensitivity and specificity than contrast-enhanced CT in the revelation of distant metastases of breast cancer; the sensitivity of PET/CT examinations was $100 \%$, specificity was $80 \%$, and positive and negative predictive values were $90.9 \%$ and $100 \%$, respectively, while the sensitivity of CT alone was 95\%, specificity was $80 \%$, and positive and negative predictive values were $90.4 \%$ and $88.8 \%$, respectively, in detected lymph node metastasis. Two lymph nodes were false positive by $\mathrm{PET} / \mathrm{CT}$; reason for false positive was positive axillary lymph nodes that presented total resolution in a progressed case on a follow-up study, denoting its benign/inflammatory nature.

The sensitivity of PET/CT examinations in detected bone lesion deposits was $91.6 \%$, specificity was $100 \%$, positive and negative predictive values were $100 \%$ and $94.7 \%$, respectively, while the sensitivity of CT alone was $75 \%$, specificity was $94.4 \%$, and positive and negative predictive values were $90 \%$ and $85 \%$, respectively. One bone lesion was false negative by PET/CT. The reason for false negative was due to low SUV max lesion (in the second follow-up study, the lesion demonstrate increase FDG uptake).

The sensitivity of PET/CT examinations in detected pulmonary nodules metastasis was $100 \%$ and specificity was $100 \%$ while the sensitivity of CT alone was $72.2 \%$ and specificity was $73.6 \%$.

The sensitivity of PET/CT examinations in detected hepatic deposits was $80 \%$, specificity was $100 \%$, and positive and negative predictive values were $100 \%$ and $96 \%$ respectively, while the sensitivity of CT alone 


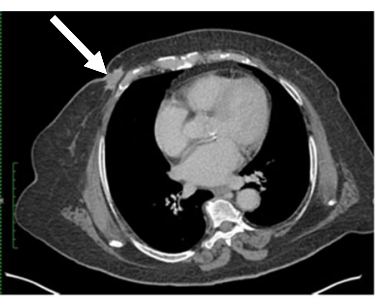

(A1) CECT

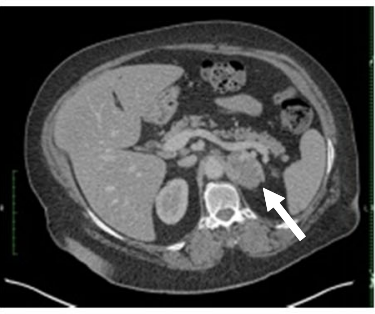

(B1) CECT

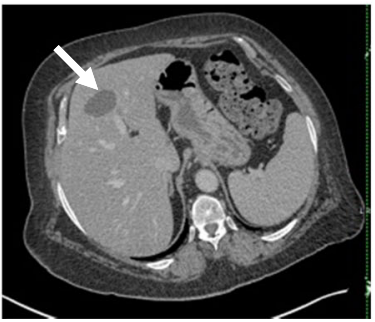

(C1) CECT

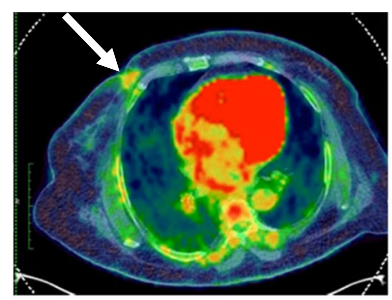

(A2) Fused PET/CT

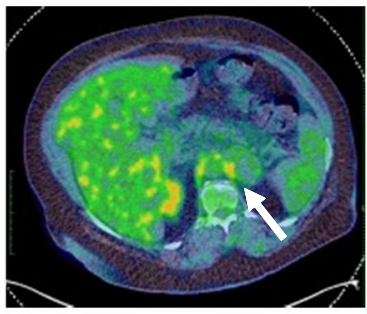

(B2) Fused PET/CT

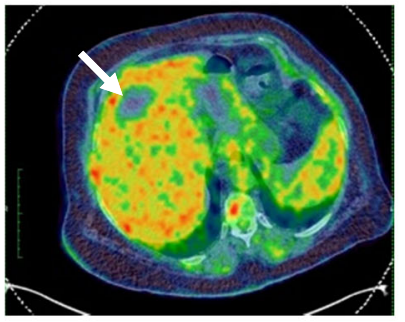

(C2) Fused PET/CT

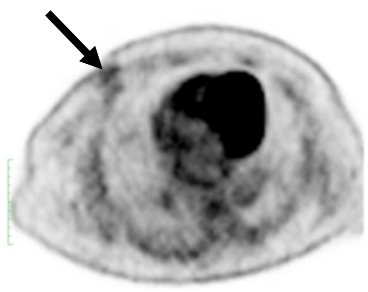

(A3) PET

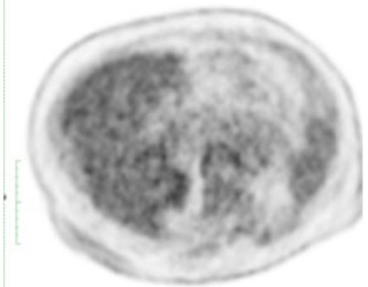

(B3) PET

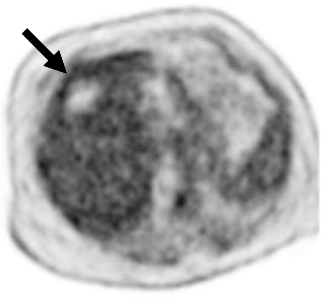

(C3) PET

Fig. 2 a $(\mathbf{1}, \mathbf{2}, \mathbf{3}) C E C T$, fused PET CT, and PET axial images show increase FDG uptake corresponding to lower inner quadrant enhancing mass with speculated margin, the lesion extends to the adjacent skin. It measures $3.7 \times 1.8 \mathrm{~cm}$ with SUVmax 2.8. b (1, 2, 3) CECT, fused PET CT, and PET axial images show mild marginal increase FDG uptake corresponding to left adrenal mass lesion measuring $5 \times 3.7 \mathrm{~cm}$ with SUVmax 2.8 (false negative result). c (1, 2, 3) CECT, fused PET CT, and PET axial images show hypodense, non-enhanced segment IV hepatic lesion with no FDG up take. Cystic lesion measuring $4.4 \times 2.8 \mathrm{~cm}$. Impression: Metabolically active right breast neoplasm. Mild metabolically active left adrenal mass, possible metastasis. Left hepatic lobe cystic lesion with no FDG uptake

was $40 \%$, specificity was $84 \%$, and positive and negative predictive values were $33.4 \%$ and $87.5 \%$, respectively. One hepatic lesion was false negative by PET/ CT. The reason for false negative was due to low SUV max lesion (in the second follow-up study, the lesion demonstrate increase FDG uptake and increase in size).

The sensitivity of PET/CT examinations in detected other visceral metastasis was $88.8 \%$, specificity was $90.4 \%$, and positive and negative predictive values

Table 3 Sensitivity, specificity, accuracy, and positive and negative predictive values of FDG-PET/CT and contrastenhanced $C T$ in all breast lesions cases

\begin{tabular}{llllll}
\hline Breast lesion & Sensitivity & Specificity & PPV & NPV & Accuracy \\
\hline PET/CT & $100.0 \%$ & $95.45 \%$ & $88.89 \%$ & $100.0 \%$ & $96.7 \%$ \\
CT & $81.25 \%$ & $90.41 \%$ & $76.47 \%$ & $93.02 \%$ & $88.33 \%$ \\
\hline
\end{tabular}

PPV positive predictive value, NPV negative predictive value were $80 \%$ and $95 \%$, respectively, while the sensitivity of CT alone was 55.5\%, specificity was $95.2 \%$, and positive and negative predictive values were $83 \%$ and $83 \%$, respectively. One adrenal lesion was false negative and another two lesions detected at adrenal and ovarian were false positive by PET/CT. Reason for false negative adrenal lesion was low SUV max (by complementary MRI, no signal drop and increase SUV max was seen in the second follow-up study) and the reason for false positive of the other adrenal nodule was high SUV max in the lesion (complementary chemical shift MRI demonstrate signal drop in out phase images confirmed it is lipid-rich adenoma) while the reason of the other false positive ovarian lesion was high SUV on a follow-up study (the lesion disappeared with no therapy, this findings represented an inflammatory process).

According to this result the fused PET/CT examination shows high sensitivity and specificity in 
Table 4 Diagnostic rendering of PET/CT and contrast-enhanced CT in 30 patients with breast cancer to detect distant metastasis

\begin{tabular}{|c|c|c|c|c|c|c|c|c|}
\hline & \multicolumn{4}{|c|}{ LN No (30) } & \multicolumn{4}{|c|}{ Bone No (30) } \\
\hline & \multicolumn{2}{|c|}{ Cancer $(n=20)$} & \multicolumn{2}{|c|}{ Non cancer $(n=10)$} & \multicolumn{2}{|c|}{ Cancer $(n=12)$} & \multicolumn{2}{|c|}{ Non cancer $(n=18)$} \\
\hline & TP & FN & FP & TN & TP & FN & FP & TN \\
\hline PET/CT & 20 & 0 & 2 & 8 & 11 & 1 & 0 & 18 \\
\hline \multirow[t]{4}{*}{ CT } & 19 & 1 & 2 & 8 & 9 & 3 & 1 & 17 \\
\hline & \multicolumn{4}{|c|}{ Lung No (30) } & \multicolumn{4}{|c|}{ Liver No (30) } \\
\hline & \multicolumn{2}{|c|}{ Cancer $(n=11)$} & \multicolumn{2}{|c|}{ Non cancer $(n=19)$} & \multicolumn{2}{|c|}{ Cancer $(n=5)$} & \multicolumn{2}{|c|}{ Non cancer $(n=25)$} \\
\hline & TP & FN & FP & TN & TP & FN & FP & TN \\
\hline PET/CT & 11 & 0 & 0 & 19 & 4 & 1 & 0 & 25 \\
\hline \multirow[t]{4}{*}{ CT } & 8 & 3 & 5 & 14 & 2 & 3 & 4 & 21 \\
\hline & \multicolumn{8}{|c|}{ Other sites No (30) } \\
\hline & \multicolumn{2}{|c|}{ Cancer $(n=9)$} & \multicolumn{2}{|c|}{ Non cancer $(n=21)$} & & & & \\
\hline & TP & FN & FP & TN & & & & \\
\hline PET/CT & 8 & 1 & 2 & 19 & & & & \\
\hline CT & 5 & 4 & 1 & 20 & & & & \\
\hline
\end{tabular}

$T P$ true positive, $F N$ false negative, $F P$ false positive, $T N$ true negative

detecting lymph nodes, bone, pulmonary, hepatic, and other visceral metastasis in cases of breast cancer comparing to the CT study alone. The diagnostic power of PET/CT in our study is in line with the study of Abo-Sheisha et al. [13] which included 50 patients with suspected distant metastasis. The sensitivity, specificity, and positive and negative predictive values of PET/CT were respectively 97\%, 93\%, 97\%, and $93 \%$ while the conventional imaging procedures

Table 5 Sensitivity, specificity, accuracy, and positive and negative predictive values of FDG-PET/CT and contrastenhanced CT in lymph nodes, bone, lung, liver, and other visceral metastasis

\begin{tabular}{|c|c|c|c|c|c|}
\hline & Sensitivity & Specificity & PPV & NPV & Accuracy \\
\hline \multicolumn{6}{|l|}{ LN } \\
\hline $\mathrm{PET} / \mathrm{CT}$ & $100.0 \%$ & $80.0 \%$ & $90.91 \%$ & $100.0 \%$ & $93.33 \%$ \\
\hline$C T$ & $95.0 \%$ & $80.0 \%$ & $90.48 \%$ & $88.89 \%$ & $90.0 \%$ \\
\hline \multicolumn{6}{|l|}{ Bone } \\
\hline $\mathrm{PET} / \mathrm{CT}$ & $91.67 \%$ & $100.0 \%$ & $100.0 \%$ & $94.74 \%$ & $96.67 \%$ \\
\hline$C T$ & $75.0 \%$ & $94.44 \%$ & $90.0 \%$ & $85.0 \%$ & $86.67 \%$ \\
\hline \multicolumn{6}{|l|}{ Lung } \\
\hline $\mathrm{PET} / \mathrm{CT}$ & $100.0 \%$ & $100.0 \%$ & $100.0 \%$ & $100.0 \%$ & $100.0 \%$ \\
\hline CT & $72.73 \%$ & $73.68 \%$ & $61.54 \%$ & $82.35 \%$ & $73.33 \%$ \\
\hline \multicolumn{6}{|l|}{ Liver } \\
\hline $\mathrm{PET} / \mathrm{CT}$ & $80.0 \%$ & $100.0 \%$ & $100.0 \%$ & $96.15 \%$ & $96.67 \%$ \\
\hline$C T$ & $40.0 \%$ & $84.0 \%$ & $33.33 \%$ & $87.50 \%$ & $76.67 \%$ \\
\hline \multicolumn{6}{|c|}{ Other sites } \\
\hline $\mathrm{PET} / \mathrm{CT}$ & $88.89 \%$ & $90.48 \%$ & $80.0 \%$ & $95.0 \%$ & $90.0 \%$ \\
\hline $\mathrm{CT}$ & $55.56 \%$ & $95.24 \%$ & $83.33 \%$ & $83.33 \%$ & $83.33 \%$ \\
\hline
\end{tabular}

PPV positive predictive value, NPV negative predictive had a sensitivity of $75 \%$, and a specificity of $73 \%$, positive predictive value $72 \%$ and negative predictive value $76 \%$.

The limitations of this study include the small number of patients and the biopsy findings were not available for some patients with suspected distant metastases.

\section{Conclusion}

This study strongly supports the conclusions of previous studies that have shown that PET/CT is more rigorous than $\mathrm{CT}$ alone for the revelation of breast lesions and distant metastases in breast cancer patients.

\section{Abbreviations}

PET: Positron emission tomography; CT: Computed tomography;

SUV: Standardized uptake value; n: Number; FDG: Fluorodeoxyglucose;

MRI: Magnetic resonance image

Acknowledgements

There is no acknowledgement.

Authors' contributions

$\mathrm{MS}, \mathrm{ZA}, \mathrm{DH}$, and $\mathrm{MH}$ contributed equally to study design, data collection, analysis, and interpretation of results. All authors read and approved the final manuscript.

Funding

There is no funding

Availability of data and materials

Data will be available upon request via contacting the corresponding author.

Ethics approval and consent to participate

This study was approved by the Research Ethics Committee of the National Liver Institute (NLI), Menoufia University on January 12, 2019; reference number of approval (eNLI IRB Protocol Number) 00183/2019. All patients included in this study gave written informed consent to participate in this research. 


\section{Consent for publication}

All patients included in this research gave written informed consent to publish the data contained within this study. If the patient was less than 16 years old, decrease, or unconscious when consent for publication was requested, written informed consent for the publication of this data was given by their parent or legal guardian.

\section{Competing interests}

The authors declare that they have no competing interests.

\section{Author details}

${ }^{1}$ Department of Radiodiagnosis, Faculty of Medicine, Menoufia University, shibin el kom, menoufia goverment 32511, Egypt. ${ }^{2}$ Department of Radiodiagnosis, National Liver Institute, Menoufia University, shibin el kom, menoufia goverment 32511, Egypt.

Received: 19 December 2019 Accepted: 26 June 2020

Published online: 09 July 2020

\section{References}

1. Ferlay J, Shin HR, Bray F et al (2010) Estimates of worldwide burden of cancer in 2008: GLOBOCAN 2008. Int J Cancer 127(12):2893-2917

2. Lacey JV Jr, Kreimer et al (2009) Breast cancer epidemiology according to recognized breast cancer risk factors in the prostate, lung, colorectal and ovarian (PLCO) cancer screening trial cohort. BMC Cancer 9:84

3. Shin KM, Kim HJ, Jung SJ et al (2015) Department of Radiology, Kyungpook National University Medical Center. J Breast Cancer 18(1):73-79

4. Koolen B, Vogel W, Vrancken P et al (2012) Molecular imaging in breast cancer: from whole-body PET/CT to dedicated breast PET. J Oncol:1-3

5. Kumar $\mathrm{R}$, Chauhan A, Zhuang $\mathrm{H}$ et al (2006) A. Clinicopathologic factors associated with false negative FDG-PET in primary breast cancer. Breast Cancer Res Treat 98:267-274

6. Christopher R, Elina S, Maxine J et al (2014) Retrospective analysis of 18FFDG PET/CT for staging asymptomatic breast cancer patients younger than 40 years. J Nucl Med 55(10):1578-1583

7. Dirisamer A, Halpern BS, Flory D et al (2010) Integrated contrast-enhanced diagnostic whole-body PET/CT as a first-line restaging modality in patients with suspected metastatic recurrence of breast cancer. Eur J Radiol 73:294299

8. Piva R, Ticconi F, Ceriani $V$ et al (2017) Comparative diagnostic accuracy of 18F-FDG PET/CT for breast cancer recurrence. Breast Cancer 9:461

9. Poodt IGM, Schipper R-J, de Greef BTA et al (2019) Screening for distant metastases in patients with ipsilateral breast tumor recurrence: the impact of different imaging modalities on distant recurrence-free interval. Breast Cancer Res Treat 175:419-428

10. Lim HS, Yoon W, Chung TW et al (2007) FDG PET/CT for the detection and evaluation of breast diseases: usefulness and limitations. Radiographics 27(supp| 1):S197-S213

11. Wafaie A, Kassem $H$, Kotb M et al (2014) Evaluation of the efficiency of FDG PET/CT in detection and characterization of skeletal metastases. Egypt J Radiol Nucl Med 45(1):181-190

12. Eubank WB, Mankoff DA, Vesselle HJ et al (2002) Detection of locoregional and distant recurrences in breast cancer patients by using FDG PET. Radiographics. 22(1):5-17

13. Abo-Sheisha DM, Badawy ME (2014) The diagnostic value of PET/CT in recurrence and distant metastasis in breast cancer patients and impact on disease free survival. Egypt J Radiol Nucl Med 45(4):1317-1324

\section{Publisher's Note}

Springer Nature remains neutral with regard to jurisdictional claims in published maps and institutional affiliations.

\section{Submit your manuscript to a SpringerOpen ${ }^{\circ}$ journal and benefit from:}

- Convenient online submission

- Rigorous peer review

- Open access: articles freely available online

- High visibility within the field

- Retaining the copyright to your article

Submit your next manuscript at $\boldsymbol{\nabla}$ springeropen.com 